\title{
Endoscopic spectrum and practical classification of small bowel gastrointestinal stromal tumors (GISTs) detected during double-balloon enteroscopy
}

(잉 $\odot$

\author{
Authors \\ Institutions \\ 1 Basil Hirschowitz Endoscopic Center of Excellence, \\ University of Alabama at Birmingham, Alabama, United \\ States \\ 2 Department of Gastroenterology, Ameos University \\ teaching Hospital, Halberstadt, Germany \\ 3 Department of Gastroenterology, Mayo Clinic \\ Jacksonville, Florida, United States \\ 4 Department of Surgery, University of Alabama at \\ Birmingham, Alabama, United States \\ 5 University of Belgrade, Belgrade, Serbia
}

Alvaro Martinez-Alcalá', Lucía C. Fry¹,2, Thomas Kröner ${ }^{3}$, Shajan Peter ${ }^{1}$, Carlo Contreras ${ }^{4}$, Klaus Mönkemüller ${ }^{1,2,5}$

\section{ABSTRACT} to present a practical classification. secutive case series of patients with small bowel GIST. submitted 13.11 .2018

accepted after revision 30.11 .2020

\section{Bibliography}

Endosc Int Open 2021; 09: E507-E512

DOI 10.1055/a-1341-0404

ISSN 2364-3722

(C) 2021. The Author(s).

This is an open access article published by Thieme under the terms of the Creative Commons Attribution-NonDerivative-NonCommercial License, permitting copying and reproduction so long as the original work is given appropriate credit. Contents may not be used for commercial purposes, or adapted, remixed, transformed or built upon. (https://creativecommons.org/licenses/by-nc-nd/4.0/)

Georg Thieme Verlag KG, Rüdigerstraße 14,

70469 Stuttgart, Germany

Corresponding author

Prof. Dr. med. habil. Klaus Mönkemüller, MD, PhD, FASGE,

EndoCollab, Dennig 18, 96317 Friesen, Germany

Fax: +49 7702 98-6779

moenkemueller@yahoo.com
Background and study aims Information about the endoscopic characterization of small bowel gastrointestinal tumors (GISTs) is limited. The aim of this case study was to describe the endoscopic spectrum of small bowel GISTs and

Patients and methods Observational, retrospective, con-

Results A total of 10 small bowel GISTs were found in patients ( 6 male, 4 female, mean age 52 years, range 28 to 68).). All patients presented with obscure gastrointestinal bleeding (overt, $n=8$, occult, $n=2$ ). Most GISTs were present in the proximal or middle small bowel $(n=7)$. The endoscopic tumor characteristics could be categorized as follows: submucosal round $(n=4)$, submucosal sessile $(n=2)$, and invasive/penetrating) $(n=4)$. The mucosa overlying the tumor was normal $(n=4)$, grooved $(n=3)$ or frankly ulcerated $(n=3)$. Tumor size ranged from $8 \mathrm{~mm}$ to $50 \mathrm{~mm}$. Biopsy was negative in all patients with normal mucosa but showed tumor in all patients with ulcerations. Regardless of biopsy results, all patients were sent for surgery. Nine resections were carried out. One patient refused surgery. There were no complications of endoscopy in this cohort. Conclusion Our series shows that GISTs have a wider spectrum of endoscopic characteristics than previously described. The round type with normal overlying mucosa was equally prevalent as the grooved or ulcerated variant. Endoscopists should be aware of this wide spectrum of presentation of small bowel GIST.

\section{Introduction}

Gastrointestinal stromal tumors (GIST) are mesenchymal neoplasms that arise from the cells of Cajal in the gastrointestinal tract $[1,2]$. While most GISTs are located in the stomach, $30 \%$ of GISTs are found in the small bowel [2,3]. Small-bowel GISTs tend to be more aggressive and have a worse prognosis with a 5 -year survival rate of $25 \%$ [1-3]. Therefore, an early diagnosis is mandatory. Patients with GIST usually present with obscure gastrointestinal bleeding, which results from a surface ulceration and necrosis [4-6]. Other complications of GISTs include bowel obstruction, intussusception, and tumor perforation [36]. The diagnosis of small bowel GIST can be very difficult [4-7]. Because of its non-specific initial symptoms, patients are often diagnosed with a small bowel GIST only in advanced, metasta- 
sized stages of the disease, when curative therapeutic option is not possible [2-7].

Whereas several studies have described the radiologic appearance of small bowel GIST, there are very limited data on the endoscopic appearance of this tumor [4, 5]. Older radiological studies showed that submucosal GISTs appear as smooth, round filling defects that may or may not ulcerate $[8,9]$. Subserosal GISTs are extrinsic or exocentric masses that displace adjacent bowel loops. Intraluminal GISTs may be hypervascular and cause hemorrhage and ulceration [4-6]. On computed tomography (CT), GISTs present as a mass that can be smooth, irregular or lobulated in appearance [8, 9]. Pathology assessment should include immunohistochemical staining for KIT and possibly DOG1 expression, and mutational analysis can have prognostic and predictive value for certain patients [1-3,5].

The endoscopic characteristics of small bowel GISTs are not widely described. Indeed, there are only limited reports describing small bowel GISTs. Furthermore, most reports lack endoscopic images of small bowel GISTs. Thus, the aims of this study were to describe the endoscopic spectrum of small bowel GISTs and to organize the characteristics of GIST based on the endoscopic appears.

\section{Patients and methods}

This was a single-center, observational, retrospective, consecutive case series of patients with small bowel GIST. Double-balloon enteroscopy (DBE) was performed between June 2013 and May 2016 in a collective of patients for whom information was included in a prospective database. Conduct of the study was in accordance with the Helsinki Declaration and Institutional Review Board approval (UAB Protocol Number X130611008).

The inclusion criteria were: adult patients $>18$ years with a verified GIST of the small bowel detected during deep enteroscopy. Exclusion criteria included patients $<18$ years, pregnancy, and inability to undergo DBE. Inability to undergo DBE was defined as those patients with high anesthesia risk. All the patients provided written informed consent to undergo DBE.

DBE was performed using a therapeutic Fujinon enteroscope (EN-450 T5/20, Fujinon Corp., Omiya, Japan). The depth of scope insertion was calculated based on the method described by May et al. [10]. Small-bowel cleansing was done the day before the procedure using $4 \mathrm{~L}$ of a standard colon lavage solution. The procedure was performed under general anesthesia as it is standard at the University of Alabama at Birmingham. Biopsies were performed in all cases where a tumor or protrusion of the small bowel could be detected using a standard forceps. At least six biopsies were obtained from each lesion. In cases of lesions with normal underlying mucosa we perform the "biopsyon-biopsy" technique. Injection with India-ink (Spot Ink, Ohio, United States) was performed to demark the tumor site in order to facilitate intraoperative detection of the primary tumor. Small bowel biopsies were fixed and preserved in $10 \%$ formalin for histopathological and immunohistochemical evaluation. Special stains for hematoxylin \& eosin features and antibody panel including CD117, CD34, desmin, smooth muscle actin, S-100 protein, and Ki67 were performed. All procedures were

\section{Endoscopic GIST Classification}

\begin{tabular}{|c|c|c|}
\hline Size & Growth & Mucosal Surface \\
\hline $\mathrm{S} 1<10 \mathrm{~mm}$ & G1 Round/oval & M1 Normal villi \\
\hline S2 $10-20 \mathrm{~mm}$ & G2 Sesil & $\begin{array}{l}\text { M2 Slot/umbilica } \\
\text { defects }\end{array}$ \\
\hline $\mathrm{S} 3>20 \mathrm{~mm}$ & G3 Invasive & M3 Ulcerate \\
\hline
\end{tabular}

a

\section{Growth}

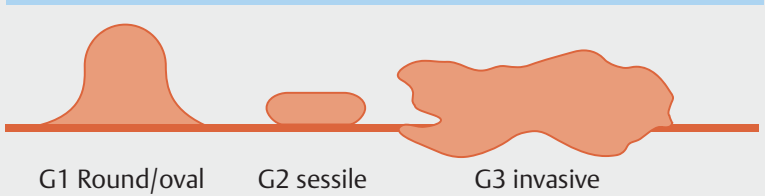

Mucosal surface

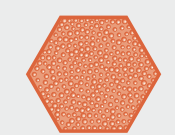

M1-Normal villi

b

Fig. 1 GMS (growth, mucosa and size) classification of small bowel gastrointestinal stromal tumor (GIST). performed by two therapeutic endoscopists (KM and SP), who had ample experience performing DBE (at least previous 700 DBE procedures each). The following information was collected: patient characteristics, laboratory data, procedure time, description of all the lesions, at least 10 pictures of each lesion, at least six biopsies of each case, location of the lesion, indication, procedure time, instruments used, complications, and follow-up.

A suspicious GIST was defined as the presence of a yellowish submucosal tumor covered either by normal appearing mucosa or with signs of ulceration or compression from outside the small bowel lumen. A definitive diagnosis was defined as histological confirmation of a GIST. Careful inspection and characterization of the small bowel GIST was performed. We defined the endoscopic characteristics based on shape, mucosal surface, and size ( $>$ Fig. 1 ).

Patient characteristics and endoscopic and histological findings as well as diagnostic and therapeutic outcomes are presented using descriptive statistics with means and ranges.

\section{Results}

A total of 12 DBEs were performed in 10 patients with small bowel GISTs during a four-year period (6 male, 4 female) ( $\triangleright$ Table 1 ). The mean age of the patients was 52 years (range 28 to 68). Mean duration of DBE was of 85 minutes (range 35 to 125 min). All patients presented with obscure gastrointestinal bleeding (overt, $n=8$, occult, $n=2$ ). There were no complications of related endoscopy in this cohort. Seven patients had a 
- Table1 Demographic, clinical, endoscopic, histologic and therapeutic characteristic of the study group.

\begin{tabular}{|c|c|c|c|c|c|c|c|c|c|}
\hline $\mathrm{Nr}$ & Age & Sex & OGIB & CE & СTA & DBE & Mucosa & Biopsies & Surgery \\
\hline 1 & 56 & M & Overt & Pos & Pos & Oral & Normal & Negative & Yes \\
\hline 2 & 62 & $\mathrm{~F}$ & Occult & $\mathrm{Neg}$ & Pos & Oral & Grooved & Positive & Yes \\
\hline 3 & 28 & $\mathrm{M}$ & Overt & ND & ND & $\mathrm{O} / \mathrm{A}$ & Ulcerated & Positive & Yes \\
\hline 4 & 49 & $M$ & Overt & Pos & $\mathrm{Neg}$ & Oral & Normal & Negative & Yes \\
\hline 5 & 52 & $\mathrm{~F}$ & Overt & ND & ND & Anal & Ulcerated & Positive & No \\
\hline 6 & 57 & M & Occult & $\mathrm{Neg}$ & $\mathrm{Neg}$ & $\mathrm{O} / \mathrm{A}$ & Normal & Negative & Yes \\
\hline 7 & 42 & M & Overt & Pos & Pos & Oral & Grooved & Positive & Yes \\
\hline 8 & 68 & $\mathrm{~F}$ & Overt & Pos & $\mathrm{Neg}$ & Oral & Normal & Negative & Yes \\
\hline 9 & 47 & $\mathrm{~F}$ & Overt & $\mathrm{Neg}$ & $\mathrm{Neg}$ & Oral & Ulcerated & $\mathrm{Nd}$ & Yes \\
\hline 10 & 59 & M & Overt & ND & ND & Oral & Grooved & Positive & Yes \\
\hline
\end{tabular}

CE, capsule endoscopy; pos, positive; neg, negative; OGIB, obscure gastrointestinal bleeding; ND, not done; CT, computed tomography of the abdomen; DBE, double balloon enteroscopy; O, oral; A, anal.

previous capsule endoscopy study, which was negative in three. Most GISTs were present in the proximal or middle small bowel $(n=7)$. Two patients had the tumor in the distal jejunum. In one patient the actively bleeding GIST was located in the ileum, about $50 \mathrm{~cm}$ proximal to the ileocecal valve. CT showed a large mesenteric tumor in one patient (with a huge necrotic small bowel tumor). CT was performed in a total of seven patients and could not detect the tumor in four patients. The endoscopic tumor characteristics could be categorized as follows: submucosal round $(n=4)$, submucosal sessile $(n=2)$, and invasive/penetrating) $(n=4)$. The mucosa overlying the tumor was normal $(n=4)$, grooved $(n=3)$ or frankly ulcerated $(n=3)$. Biopsy was negative in all patients with normal mucosa $(n=4)$ but showed tumor in all patients with ulcerations $(n=3)$ and two with grooved appearance. In one patient with active bleeding and small ulceration no ulcers were performed. Therefore, the diagnostic yield of endoscopic biopsy was $55 \%$. Regardless of biopsy results, all patients were sent for surgery. Nine resections were carried out. One patient refused surgery. This patient had an ulcerated lesion and a histologic diagnosis of GIST. After interdisciplinary conference tumor conference, a decision was reached to offer the patient therapy with imatinib, which the patient accepted. On latest follow-up at 7 months the patient was stable and without further bleeding.

The endoscopic spectrum of GIST is presented in > Fig. 2 , >Fig. 3, > Fig. 4, > Fig. 5, > Fig. 6, and > Fig. 7. The endoscopic classification is primarily based on growth (G), appearance of the mucosa (M) and size of the lesion (S), "GMS classification". Based on endoscopic findings we observed that GIST can growth (G) in different ways, as round or oval (type G1) (\ Fig. 2a, > Fig.3a, > Fig.3b), sessile (type G2) ( Fig.3c, > Fig.3d, > Fig.4), invasive (type G3) (> Fig.4, > Fig.5). Based on mucosal (M) appearance the GIST could be classified as normal (intact mucosa) (M1) ( Fig. 2, > Fig.3), with the classical appearance of slot or umbilical defect (M2) (ฉ Fig.4, \ Fig.6), and ulcerative mucosal surface (M3) ( $\triangleright$ Fig.5). We also classified GIST
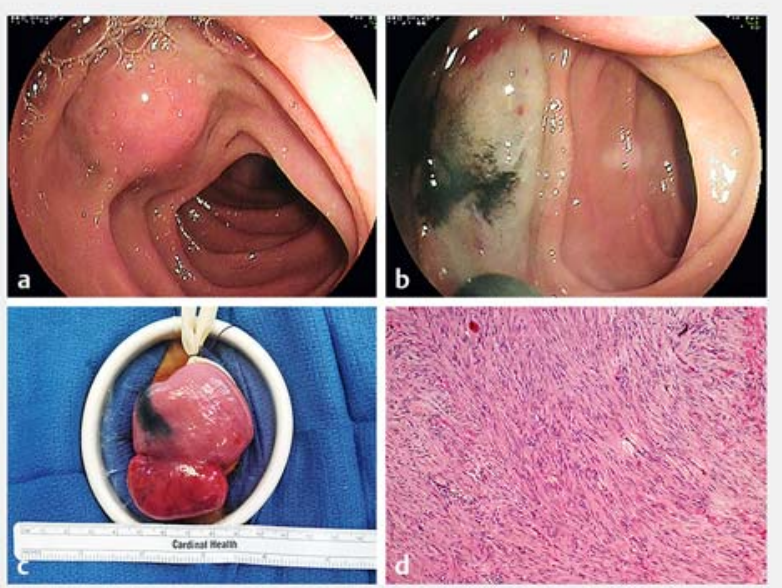

- Fig. 2 Typical presentation of small bowel GIST in our study. a Round submucosal tumor mimicking a lipoma (G1), with intact mucosa (M1). b Injection of submucosal ink to mark the site. c Minimally invasive surgery to resect the GIST. The ink marking assisted the surgeon in finding the lesion. $\mathbf{d}$ Histology of a typical GIST.

according to the size (S) of the lesion; <10 mm (S1) ( Fig. 1),

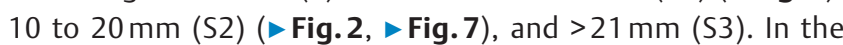
theoretical case of presence of the afferent vessel of the lesion, would be add $\mathrm{A} 1$ to the previous classification ( $\mathbf{F i g . 4 d , ~}>$ Fig. 7). In total there are three different endoscopic characteristics of each GIST (size, growth and mucosal surface) with the addition of type $\mathrm{A} 1$ when the special feature of the afferent vessel is found. 

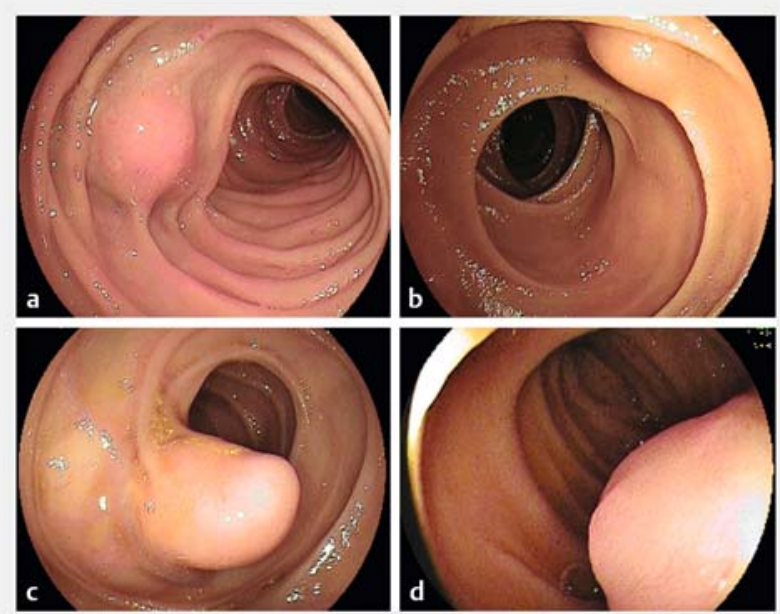

- Fig. 3 Spectrum of protruded GISTs. a Round submucosal lesion (G1) with intact mucosa (M1), 10-20 mm in size (S2). b Small, oval, yellowish, submucosal lesion (G1) with intact mucosa (M1), measuring $<10 \mathrm{~mm}(\mathrm{~S} 1)$. This lesion may be easily missed by the inattentive eye or inexperienced endoscopist. c Sessile, protruding submucosal mass with intact mucosa (G2, M1, S2). d Sessile, protruding mass without ulcerations measuring 16 to $20 \mathrm{~mm}$ (G2, M3, S2).
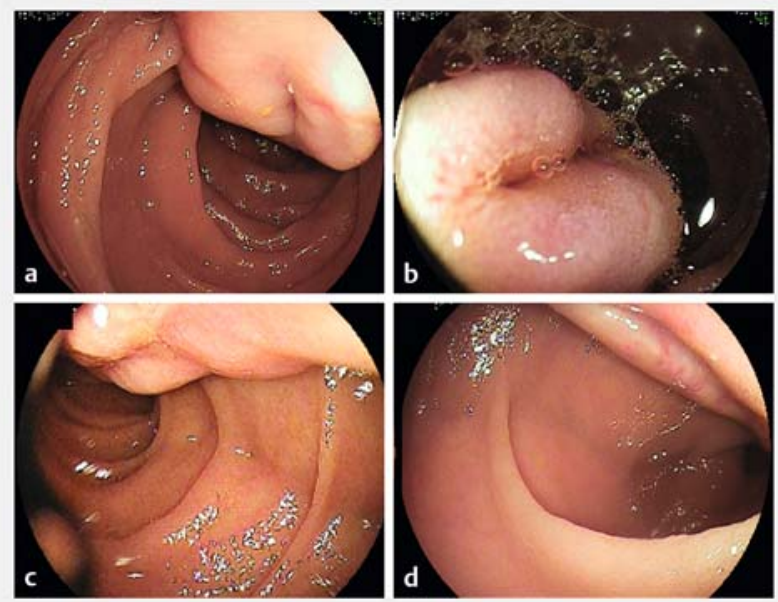

Fig. 4 "Typical" umbilicated or grooved lesion in laterally spreading GIST (G3, M2). a This "slit", "umbilicus," or "groove" has been considered a "typical appearance" of GIST (M2). However, only $40 \%$ of our patients presented with this type of endoscopic appearance. $\mathbf{b}$ Laterally growing lesion with umbilicated and congested mucosa (G2M2). c Laterally growing, sessile lesion with partial grove (G2M2). d Groove with mucosal hypervascularity (G2).
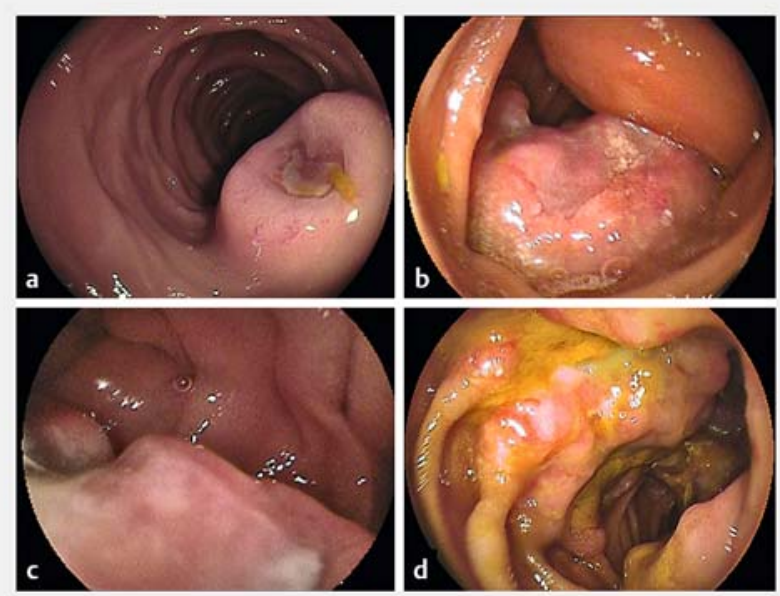

- Fig. 5 Endoscopic spectrum of ulcerated GIST. a Sessile lesion with ulceration measuring $10-20 \mathrm{~mm}(\mathrm{G} 2, \mathrm{M} 3, \mathrm{S2})$. B. Large, laterally spreading, ulcerated GIST measuring $>21 \mathrm{~mm}(\mathrm{G} 3, \mathrm{M} 3, \mathrm{~S} 3)$. C. Large, laterally spreading GIST (G3, M3, S3). D. Invasive GIST with hemicircumferential luminal involvement and partial stenosis $(G 3, M 3, S 3)$.
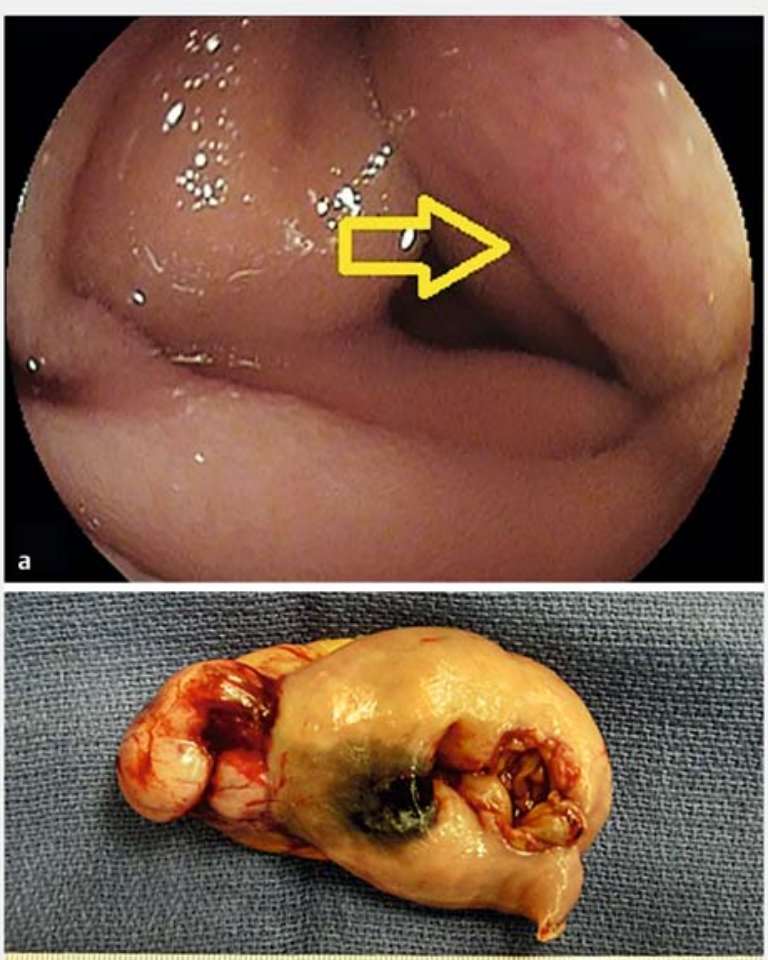

MEDUNE

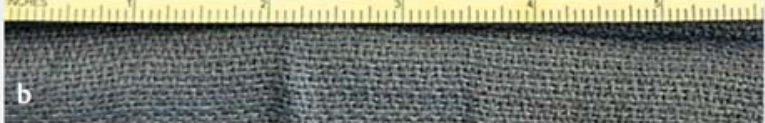

Fig. 6 Lumen-occluding GIST. a Groove-type lesion measuring $>21 \mathrm{~mm}$ (G2, M2, S3). b Surgical specimen. 


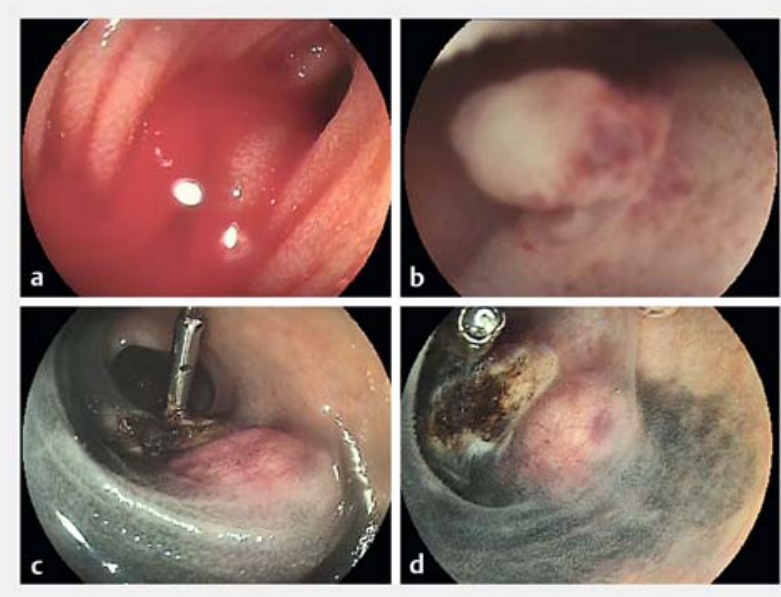

Fig. 7 Massively bleeding small bowel GIST. a This patient was transferred from another hospital with massive hematochezia. Fresh blood was coming from the ileum. $\mathbf{b}$ A bleeding lesion could be found approximately $50 \mathrm{~cm}$ proximal to the ileocecal valve. c Successful hemostasis of a bleeding vessel (a1) feeding this small, yellowish, oval lesion (G1, M1, S1, A1).

\section{Discussion}

To the best of our knowledge, this is one of the largest series of small bowel GISTs documented by DBE. We systematically described the endoscopic appearance of small bowel GIST. In this study we demonstrated that endoscopic classification of GIST is feasible and simple, and in addition, we showed that small bowel GIST has a wider endoscopic spectrum than previously thought, as most reports and series depict these tumors with an umbilicated or grooved surface. The round type with normal overlying mucosa was equally prevalent as the grooved or ulcerated variant.

During DBE we observed that small bowel GIST had specific appearances that may allow it to be classified based on endoscopic findings. In only $30 \%$ of our cases, small bowel GIST presented with the classical description of oval or round growth inside the lumen (G2). The key characteristics of small bowel GIST were its sessile and protruding shape. This is important to know, as capsule endoscopy and traditional enteroscopy with biopsies may be falsely negative [7,10-12]. Interestingly, some lesions mimicked lipomas (> Fig.1, > Fig.2), with a round, submucosal shape and yellowish center. None of these lesions could be diagnosed as GIST with biopsy, and it was only our previous experience with small bowel GISTs that prompted us to insist on surgical exploration and treatment of these patients [13]. This series also emphasizes the importance of submucosal ink marking to allow surgeons to easily find the lesion, thus allowing them to perform minimally invasive incision and laparoscopic surgery. The size of the lesion correlated with disease extension and metastasis. In addition, all ulcerated lesions were highly malignant. Some of the ulcerated GISTs mimicked adenocarcinoma or advanced lymphoma. Interestingly, even small lesions led to bleeding. We do not know how lesions with- out mucosal ulceration or umbilication bled. However, it is evident that all patients presented with obscure gastrointestinal bleeding and all possible causes had been ruled out by upper and lower endoscopy and in $60 \%$ also by capsule endoscopy. Here we want to emphasize that capsule endoscopy missed three tumors. This problem has been previously described in the literature [10-12]. Thus, a negative capsule endoscopy study should not preclude performing deep enteroscopy. We speculate that these lesions bleed and the mucosa rapidly heals or that the bleeding occurs at the level of the "feeding vessel," thus making it impossible for capsule endoscopy to detect. Indeed, one of our patients with a small yellow GIST presented with massive bleeding arising at the distal part of the lesion, at the level of a "feeding vessel" ( $\triangleright$ Fig. 7). This patient was treated with emergency DBE, with successful clipping of the vessel and bleeding site. Surgery was performed within 24 hours and a classic GIST was resected.

In our study, the yield of biopsies was $55 \%$, which is low. Nevertheless, most oncologists want to reach a preoperative diagnosis before embarking into surgery or chemotherapy. The argument is that with preoperative histological results, interdisciplinary planning is better, involving the oncologist, surgeon, and radiologist. We want to emphasize, however, that biopsy should not be a prerequisite for surgery, as most cases with intact mucosa have negative results. Avoiding surgery in these patients may result in tumor progression, rebleeding, and potentially death. Thus, our experience presented in this study show that appearance should be of clinical importance for endoscopists dealing with obscure gastrointestinal bleeding and atypical submucosal tumors.

We want to acknowledge the limitations of our study. First, all retrospective studies have inherent deficits. However, the immense collection of endoscopic images, coupled with the careful, prospective collected registry of our small bowel study group may minimize this deficit. Second, our study was small. Nevertheless, our study it is one of the largest experiences using DBE for evaluation of small bowel GIST. Furthermore, our series has the advantage of providing an extensive and detailed endoscopic description of GIST. We encourage future multicenter studies to further investigate the concepts revealed by our study. Finally, our study was carried out at a tertiary referral center. Nevertheless, any endoscopist who performs deep enteroscopy may potentially be faced with diagnosing small bowel GIST and this endoscopic study should enable a "training of the eye" for awareness of this tumor. Based on our findings, we recommend avoiding biopsies in lesions where the positivity is low or that are more likely to produce bleeding: 1) M1 lesions (smooth, normal mucosal surface and villi); 2) A1 lesions; and 3) those with visible vessels and potential bleeding risk.

\section{Conclusion}

In summary, endoscopic small bowel investigation by DBE seems to enrich the possibility of diagnosing small bowel GIST. Our study adds to the literature on the topic and we hope that the small bowel endoscopist may become sensitized to consid- 
ering the diagnosis of GIST. Our series shows that GISTs have a wider spectrum of endoscopic characteristics than previously described. Finally, this simple endoscopic classification of small bowel GIST may be a first step in providing a standard terminology to describe this puzzling tumor. Multicenter studies with a larger number of patients and a prospective design are definitely necessary to confirm our observation and to evaluate its prognostic value.

Competing interests

The authors declare that they have no conflict of interest.

References

[1] Pisters PW, Patel SR. Gastrointestinal stromal tumors: current management. J Surg Oncol 2010; 102: 530-538

[2] Joensuu H, Hohenberger P, Corless CL. Gastrointestinal stromal tumour. Lancet 2013: doi:10.1016/S0140-6736(13)60106-3

[3] Mullady DK, Tan BR. A multidisciplinary approach to the diagnosis and treatment of gastrointestinal stromal tumor. J Clin Gastroenterol 2013; 47: 578-585

[4] Almeida N, Figueiredo P, Lopes S et al. Double-balloon enteroscopy and small bowel tumors: a South-European single-center experience. Dig Dis Sci 2009; 54: 1520-1524
[5] Akyüz U, Erzin Y, Cengiz C. Diagnosis of gastrointestinal stromal tumors with double-balloon enteroscopy. Turk J Gastroenterol 2010; 21: $39-41$

[6] Fry LC, Neumann H, Kuester D et al. Small bowel polyps and tumours: endoscopic detection and treatment by double-balloon enteroscopy. Aliment Pharmacol Ther 2009; 29: 135-142

[7] Jovanovic I, Krivokapic Z, Menkovic N et al. Ineffectiveness of capsule endoscopy and total double-balloon enteroscopy to elicit the cause of obscure overt gastrointestinal bleeding: think GIST! Endoscopy 2011; 43: (Suppl. 02): E91-E92

[8] Buckley JA, Fishman EK. CT evaluation of small bowel neoplasms: spectrum of disease. Radiographics 1998; 18: 379-392

[9] Burkill G], Badran M, Al-Muderis O et al. Malignant gastrointestinal stromal tumor: distribution, imaging features, and pattern of metastatic spread. Radiology 2003; 226: 527-532

[10] Zagorowicz ES, Pietrzak AM, Wronska E et al. Small bowel tumors detected and missed during capsule endoscopy: single center experience. World J Gastroenterol 2013; 19: 9043-9048

[11] Madisch A, Schimming W, Kinzel F et al. Locally advanced small? bowel adenocarcinoma missed primarily by capsule endoscopy but diagnosed by push enteroscopy Endoscopy 2003; 35: 861-864

[12] Hakim FA, Alexander JA, Huprich JE et al. CT-enterography may identify small bowel tumors not detected by capsule endoscopy: eight year experience at Mayo Clinic Rochester. Dig Dis Sci 2011; 56: 29142919

[13] Fry LC, Bellutti M, Neumann $\mathrm{H}$ et al. Incidence of bleeding lesions within reach of conventional upper and lower endoscopes in patients undergoing double-balloon enteroscopy for obscure gastrointestinal bleeding. Aliment Pharmacol Ther 2009; 29: 342-349 